\title{
INFLUÊNCIA DO TRÁFEGO DE VEÍCULOS COMERCIAIS EM RODOVIAS VICINAIS NÃO PAVIMENTADAS: ESTUDO DE CASO APLICADO À VCS 346, VIÇOSA, MG $^{1}$
}

\author{
Taciano Oliveira da Silva², Carlos Alexandre Braz de Carvalho ${ }^{3}$, Dario Cardoso de Lima ${ }^{3}$, Maria Lúcia \\ Calijuri $^{3}$ e Carlos Cardoso Machado ${ }^{3}$
}

\begin{abstract}
RESUMO - Este artigo aborda a influência da variação da carga por eixo e da pressão de enchimento dos pneus sobre as deflexões superficiais recuperáveis previstas na estrutura de pavimento da estrada não pavimentada VCS 346, considerada representativa da malha vicinal do Município de Viçosa, MG. O programa de estudo considerou as seguintes análises: (i) contagem volumétrica do tráfego, inclusive de veículos comerciais do tipo 2C; (ii) deflexões recuperáveis previstas, em função dos dados disponíveis no estudo; (iii) estrutura do pavimento, com dados referentes ao material das camadas. Com as análises, observou-se que a aplicação de níveis mais críticos de carregamento interferiu significativamente na vida útil do pavimento e, dependendo da combinação da carga por eixo e da pressão de enchimento dos pneus, a vida útil da estrada pode sofrer redução da ordem de $89 \%$. Investigaram-se ainda, neste trabalho, dois métodos para dimensionar a espessura do revestimento primário para rodovias não pavimentadas. Um desses métodos foi o empírico, qual seja, o método do DNIT para dimensionamento de pavimentos flexíveis; e o outro foi fundamentado em teoria elástica para sistemas de camadas. Para o dimensionamento de estradas não pavimentadas melhoradas com uma camada de revestimento primário (sistema de duas camadas), concluiu-se que o procedimento mais adequado foi o emprego da teoria elástica.
\end{abstract}

Palavras-chave: Carga por eixo, Pressão de enchimento dos pneus e Desempenho de pavimento.

INFLUENCE OF TRAFFIC OF COMMERCIAL VEHICLES IN UNPAVED SECONDARY ROADS: A CASE STUDY APPLIED TO THE VCS 346, VIÇOSA, MG

\begin{abstract}
This paper approaches the influence of the variation of load per axle and tire pressure on the recoverable superficial deflections in a pavement structure of the VCS 346 unpaved road taken as representative of the secondary road net of Viçosa-MG, Brazil. The program of study considered the following analysis: (i) traffic volume counting including 2C type commercial vehicles; (ii) predicted recoverable superficial deflections in function of the available data from the study; (iii) structure of the pavement including data from the materials of its layers. By the analyses, it was verified that the application of more critical loading levels affected significantly the pavement serviceable life and depending on the combination of axle load and tire pressure, road pavement serviceable life could be reduced by $89 \%$. In this study, it was also investigated the application of two methods to measure sub-grade reinforcement layer thickness of unpaved roads. One of these two methods was empirical, the DNIT's Flexible Pavement Design criteria and the other was based on elastic theory applied to a two layer system. The use of the elastic theory was more adequate to measure unpaved roads improved with wearing courses (two layer system) proved to be the best procedure for designing unpaved road systems.
\end{abstract}

Keywords:Axle load, Tire pressure and Pavement performance.

\footnotetext{
${ }^{1}$ Recebido em 21.04.2009 e aceito para publicação em 14.04.2011.

${ }^{2}$ Universidade Federal de São João Del-Rei, UFSJ, Brasil. E-mail: <taciano@ufsj.edu.br>

${ }^{3}$ Universidade Federal de Viçosa, UFV, Brasil.E-mails: <cabraz@ufv.br>, declima@ufv.br>, <calijuri@ufv.br>e <machado@ufv.br>.
} 


\section{INTRODUÇÃO}

As estradas exercem importante papel no desenvolvimento econômico e social de um país. No Brasil, o transporte rodoviário é considerado a principal via de integração, fundamental no escoamento da produção industrial e agrícola brasileira (TRINDADE et al., 2005). A malha rodoviária nacional apresenta uma extensão da ordem de $1.725 .000 \mathrm{~km}$, com aproximadamente $10 \%$ pavimentados; desse total, $83 \%$ enquadram-se na categoria de rodovias municipais e vicinais, dos quais $1,2 \%$ se encontra pavimentado (VELTEN et al., 2006).

O tráfego comercial que solicita um pavimento é o carregamento a ser considerado nos métodos de dimensionamento, como destacou Balbo (2007), sejam eles empíricos, sejam mecanísticos. Portanto, existe uma importante necessidade de se padronizar o tráfego, pois na realidade ele se apresenta de forma bastante complexa: veículos diferentes, cargas aleatórias, várias configurações de eixo e rodas, velocidades variáveis etc. Por esses motivos, é usual representar o tráfego total e a intensidade das cargas transportadas por meio do número $(\mathrm{N})$, que representa o número equivalente de operações do eixopadrão de 8,2 t e expressa um tráfego virtual que produz o mesmo efeito destrutivo do tráfego real.

A partir de estudos desenvolvidos pela AASHTO Road Test, no final da década de 1950, determinou-se que um eixo simples de roda dupla com carga de $18.000 \mathrm{lb}$ ou $82 \mathrm{kN}(8,2 \mathrm{tf})$ e $563 \mathrm{kPa}$ (80 psi) de pressão de enchimento dos pneus seria considerado eixo-padrão. Os efeitos destrutivos de cargas quaisquer por eixo sobre pavimentos podem ser atribuídos, comparativamente, ao provocado por determinado número de passagens do eixo-padrão. Pode-se ter correlação de cada tipo de eixo ou conjunto de eixos com o eixo-padrão, por meio do Fator de Equivalência de Cargas (FEC).

As maiores críticas ao cálculo do número $\mathrm{N}$ acontecem devido aos procedimentos empíricos sobre os quais está fundamentada a determinação do FEC expresso em dano relativo à configuração de um eixopadrão de 82 kN (TIMM; NEWCOMB, 2002). Os referidos autores comentaram que pesquisas desenvolvidas em inúmeras pistas experimentais indicam que o FEC é regido pelo tipo e espessura do pavimento, conjunto de eixos, valor da carga por eixo, pressão de enchimento e condições ambientais.
Um aspecto que merece destaque é a definição de FEC para as vias vicinais brasileiras, que em geral não apresentam tratamento superficial específico e são constituídas apenas do solo local, sendo denominadas estradas de superfície não tratada. Nesse caso, segundo Yoder e Witczak (1975) o uso de material local para a construção dessas vias é a regra em vez da exceção. Portanto, é comum o emprego de um revestimento primário nessas vias, constituído de solo granular com espessura da ordem de 20 a $30 \mathrm{~cm}$ de material solto, do que resulta uma espessura de 15 a 25 cm após a compactação, de acordo com as especificações técnicas para revestimento primário de alguns departamentos brasileiros de estradas de rodagem (DERs).

Tendo em vista a significativa amplitude da malha viária não pavimentada do Brasil, que segundo a ANTT (2005) já alcança aproximadamente 1,45 milhão de quilômetros de extensão, o estudo da influência do tráfego comercial e a análise para dimensionar espessuras de camadas de revestimento primário para essas vias são relevantes, tendo em vista o papel que elas desempenham conjuntamente com as rodovias pavimentadas. Essas vias vicinais têm caráter de vias capilares de circulação de bens de consumo (cargas), o que seguramente obriga que elas possuam condições estruturais próximas das que atendem às solicitações de tráfego das vias pavimentadas.

Considerando os aspectos técnicos das vias vicinais brasileiras não pavimentadas, o objetivo deste estudo foi analisar a influência da variação dos fatores carga por eixo e pressão de enchimento dos pneus sobre as deflexões superficiais recuperáveis, com base na teoria elástica aplicada a um sistema de duas camadas para o pavimento de uma estrada vicinal no Município de Viçosa, MG.

\section{METODOLOGIA}

\subsection{Contagem volumétrica do tráfego}

A contagem volumétrica do tráfego, inclusive de veículos de carga do tipo 2C, que são os ônibus e caminhões que possuem um eixo simples de rodas simples (ESRS) e um eixo simples de rodas duplas (ESRD), foi obtida, na forma direta, na estrada vicinal VCS 346, cuja extensão é de aproximadamente 3,1km, no Município de Viçosa, MG. Essa estrada faz a ligação entre o campus da Universidade Federal de Viçosa (UFV) e a rodovia federal BR 120. Possui tráfego leve, com menos de 
50 veículos comerciais por dia, que servem para transporte de encomendas e entregas em Viçosa ou no interior do campus da UFV.

\subsection{Deflexões recuperáveis previstas}

Para dada estrutura de pavimento, representativa da malha viária não pavimentada do Município de Viçosa, MG, e estrada vicinal não pavimentada VCS 346, foram previstos valores de deflexões recuperáveis. Em função dos dados disponíveis no estudo, foi escolhida a deflexão superficial como medida do desempenho do pavimento. Para tais fins, definiram-se três níveis de carga por eixo, como a seguir:

- $C_{1}=82 \mathrm{kN}$ (8,2 tf): carga correspondente ao eixopadrão de $18.000 \mathrm{lb}$, considerada baixa.

- $C_{2}=100 \mathrm{kN}$ (10,0 tf): carga máxima permitida pela legislação brasileira para eixo simples de rodas duplas, considerada normal.

- $C_{3}=120 \mathrm{kN}$ (12,0 tf): carga 20\% acima da máxima permitida, sendo 5\% o limite de tolerância legal. Adotou-se carga 20\% acima da permitida para mostrar os danos nas estradas quando há excesso de carga nos eixos dos veículos comerciais.

Da mesma forma, foram utilizados três níveis de pressão de enchimento dos pneus, que foram escolhidos de acordo com Albano (2005) e recomendações contidas em catálogos de fabricantes de pneus, quais sejam:

- $P_{1}=563 \mathrm{kPa}$ (80 psi): pressão-padrão utilizada na pista experimental da AASHTO e normalizada para determinação da deflexão, conforme o ME 24/94 do DNER (DNER, 1994), que tem sido adotada em diversos métodos de dimensionamento de pavimentos.

- $P_{2}=633 \mathrm{kPa}(90 \mathrm{psi})$ : pressão considerada média.

- $P_{3}=703 \mathrm{kPa}$ (100 psi): pressão considerada alta, de acordo com estudo de Albano (2005) sobre efeitos dos excessos de carga sobre a durabilidade de pavimentos.

Todos os valores de deflexão recuperável $D_{i j}$ foram divididos por $D_{p p}$, que é a medida da deflexão em 0,01 mm decorrente da combinação dos níveis-padrão dos fatores carga por eixo de 82,0 kN e pressão de enchimento de $563 \mathrm{kPa}$ (80 psi). Os quocientes das referidas divisões foram elevados à potência de 5,959, de acordo com a equação 1, apresentada por Pereira (1992), obtendo-se os FEC empírico-mecanísticos correspondentes às determinações do estudo.

$$
F E C=\left(\frac{D_{i j}}{D_{p p}}\right)^{5,959}
$$

Como parâmetro para comparação com a variação dos FEC empírico-mecanísticos, calculou-se outro conjunto de valores de FEC utilizando a relação $\left(\frac{D_{i j}}{D_{p p}}\right)$ elevada ao expoente médio de 4,298, apresentado por Pereira (1992), oriundo do experimento da AASHTO para cargas por eixo de 80 a $120 \mathrm{kN}$, conforme apresentado na Tabela 5.

\subsection{Tensões verticais na superfície do subleito}

Realizou-se ainda um estudo das tensões verticais atuantes na superfície do subleito. Para a estrutura e os parâmetros de deformabilidade contidos nas Tabelas 1 e 2 e carregamentos apresentados na Tabela 3 foram obtidas as tensões verticais na superfície do subleito, por meio do programa computacional ELSYM5, cujos valores se encontram reproduzidos na Tabela 8. Calculou-se a tensão vertical admissível no subleito pela equação 2, estabelecida em 1962 por Heukelom e Klomp.

$$
\sigma_{\mathrm{v}_{\mathrm{adm}}}=\frac{0,006 \cdot \mathrm{M}_{\mathrm{R}}}{1+0,7 \cdot \log \mathrm{N}}
$$

em que $\sigma_{v_{a d m}}$ é tensão vertical admissível no topo do subleito, $M_{R}$ é módulo de resiliência do subleito em $\mathrm{kgf} / \mathrm{cm}^{2}$ e N é o número de aplicações de carga.

\subsection{Estrutura do pavimento}

A estrutura do pavimento da estrada vicinal VCS 346 está descriminada na Tabela 1.

Os dados referentes aos materiais das camadas da estrutura do pavimento da estrada vicinal VCS 346 são apresentados na Tabela 2 .

Os módulos de resiliência (Mr) contidos na Tabela 2 foram estimados por meio da equação 3, de Heukelon e Klomp (1962 ${ }^{4}$ ) apud (PINTO; PREUSSLER, 2001).

Tabela 1 - Estrutura do pavimento da estrada vicinal VCS 346.

Table 1 -Pavement structure of the secondary road VCS 346.

\begin{tabular}{ccc}
\hline Camada & Material & Espessura $(\mathrm{cm})$ \\
\hline $\begin{array}{c}\text { Revestimento } \\
\text { primário }\end{array}$ & Saibro & 20,0 \\
\hline Subleito & $\begin{array}{c}\text { ArgissoloVermelho-Amarelo } \\
\text { de comportamento laterítico }\end{array}$ & Semi-infinita \\
\hline
\end{tabular}

Revista Árvore, Viçosa-MG, v.35, n.3, p.539-545, 2011 
Tabela 2-Dados relativos ao material das camadas do pavimento analisado.

Table 2-Data from the material of the layers of the analyzed pavement.

\begin{tabular}{lccc}
\hline Camada & $\begin{array}{c}\text { Coeficiente } \\
\text { de Poisson( } \boldsymbol{\mu})\end{array}$ & $\begin{array}{c}\text { Módulo de } \\
\text { resiliência (Mr) } \\
\left(\mathbf{k g f} / \mathbf{c m}^{2}\right)\end{array}$ & $\mathbf{C B R}^{*}(\%)$ \\
\hline $\begin{array}{l}\text { Revestimento } \\
\text { Primário }\end{array}$ & 0,40 & 1.000 & 10 \\
\hline Subleito & 0,45 & 600 & 6 \\
\hline
\end{tabular}

* Os valores de CBR foram obtidos em ensaios de laboratório na energia de compactação do Proctor normal, tanto para o subleito quanto para a camada de revestimento primário.

$$
\mathrm{Mr}=100 . C B R
$$

em que: $\mathrm{Mr}=$ Módulo de resiliência $\left(\mathrm{kgf} / \mathrm{cm}^{2}\right)$.

Os coeficientes de Poisson foram fixados de acordo com os valores apresentados por Balbo (2007).

Os valores das deflexões superficiais para a determinação dos FEC empírico-mecanísticos foram obtidos por meio da utilização do programa computacional ELSYM5. O referido programa computacional é utilizado para o cálculo de tensões, deformações e deslocamentos em sistemas de camadas elástico-lineares.

\section{RESULTADOS E DISCUSSÃO}

\subsection{Número N para a rodovia vicinal VCS 346}

Considerando uma taxa anual de crescimento linear de tráfego $\mathrm{t}=3 \%$, um período de projeto $\mathrm{P}=5$ anos e o fator climático regional (FR) de 1,4, para altura média anual de chuva entre 800 e 1.500 mm, de acordo com Senço (1997), fundamentado na precipitação média anual de $1.221 \mathrm{~mm}$ para a região de Viçosa, MG, encontrou-se um valor de operações equivalentes do eixo-padrão.

\subsection{Deflexão recuperável como resposta estrutural}

Neste estudo são apresentados valores de deflexões recuperáveis, determinadas pelo programa computacional ELSYM5, em situações típicas de carregamento, conforme mostrado na Tabela 3.
Tabela 3 - Valores de deflexões superficiais recuperáveis previstas no topo do subleito, em função da carga por eixo e pressão de enchimento.

Table 3 - Values of recoverable superficial deflections in the subgrade top in function of the the axle load and tire pressure.

\begin{tabular}{cccc}
\hline Pressão de & \multicolumn{3}{c}{ Carga por eixo (kN) } \\
\cline { 2 - 4 } enchimento & 82 & 100 & 120 \\
\cline { 2 - 4 } kPa (PSI) & Deflexões & recuperáveis & $\mathbf{( 0 , 0 1} \mathbf{~ m m})$ \\
\hline $563(80)$ & 98,90 & 120,00 & 142,00 \\
$633(90)$ & 99,30 & 120,00 & 143,00 \\
$703(100)$ & 99,60 & 121,00 & 144,00 \\
\hline
\end{tabular}

\subsection{Relações entre deflexões recuperáveis}

Na Tabela 4 são apresentados os valores de FEC para cada combinação dos níveis de carga por eixo e pressão de enchimento dos pneus.

A Tabela 5 apresenta outro conjunto de valores de FEC utilizando a relação $\left(\frac{D_{i j}}{D_{p p}}\right)$ elevada ao expoente médio de 4,298, apresentado por Pereira (1992), oriundo do experimento da AASHTO para cargas por eixo de 80 a $120 \mathrm{kN}$.

Tabela 4 -Valores de FEC empírico-mecanísticos calculados. Table 4 -Calculated values of the mechanistic-empirical LEFs.

\begin{tabular}{cccc}
\hline \multirow{2}{*}{$\begin{array}{c}\text { Carga por } \\
\text { eixo }(\mathrm{kN})\end{array}$} & \multicolumn{3}{c}{ Pressão de enchimento kPa (PSI) } \\
\cline { 2 - 4 } & $563(80)$ & $633(90)$ & $703(100)$ \\
\cline { 2 - 4 } & Fator de & Equivalência de Carga (FEC) \\
\hline 82 & 1,000 & 3,166 & 8,632 \\
100 & 1,024 & 3,166 & 9,000 \\
120 & 1,043 & 3,326 & 9,382 \\
\hline
\end{tabular}

Tabela 5 - Valores de FEC empírico-mecanísticos da AASHTO, segundo Pereira (1992).

Table 5 - AASHTO mechanistic-empirical LEFs values according to Pereira (1992.

\begin{tabular}{cccc}
\hline \multirow{2}{*}{$\begin{array}{c}\text { Carga por } \\
\text { eixo }(\mathrm{kN})\end{array}$} & \multicolumn{3}{c}{ Pressão de enchimento kPa (PSI) } \\
\cline { 2 - 4 } & $563(80)$ & $633(90)$ & $703(100)$ \\
\cline { 2 - 4 } & Fator de Equivalência de Carga (FEC) \\
\hline 82 & 1,000 & 2,296 & 4,733 \\
100 & 1,017 & 2,296 & 4,878 \\
120 & 1,031 & 2,379 & 5,027 \\
\hline
\end{tabular}

\footnotetext{
${ }^{4}$ HEUKELOM, W.; KLOMP, A. J. G. Dynamic testing as a mens of controlling pavements during and after construction. Proceedings... $1^{\circ}$ Intern. Conference on the Structural Design of Asphalt Pavements Ann Arbor, University Michigan, 1962.
}

Revista Árvore, Viçosa-MG, v.35, n.3, p.539-545, 2011 
De acordo com Albano (2005), o parâmetro FEC tem sido largamente utilizado como medida de desempenho de pavimentos flexíveis porque, comparativamente, mede os efeitos que os excessos de carga por eixo provocam nessas estruturas. Nas duas situações apresentadas nas Tabelas 4 e 5, observa-se que, quanto maior o nível da carga por eixo e da pressão de enchimento dos pneus, mais representativo é o efeito destrutivo, com deflexões recuperáveis, no pavimento.

Relacionando a deflexão recuperável a partir dos FEC com a vida do pavimento, nas Tabelas 6 e 7 é mostrado o desempenho do pavimento com base na evolução dos FEC e na redução da vida útil. As previsões de redução da vida útil da estrutura do pavimento (\%) para os casos em estudo foram determinadas pela equação 4 .

$$
R V U(\%)=100-\left(\frac{F E C_{p p}}{F E C_{i j}} \cdot 100\right)
$$

em que RVU (\%) é a previsão de redução da vida útil da estrutura do pavimento, em porcentagem; FEC $_{p p}$ é o fator de equivalência de cargas referente ao eixopadrão; e $\mathrm{FEC}_{\mathrm{ij}}$ é o fator de equivalência de cargas referente a um eixo qualquer.

Tabela 6 -Evolução dos FEC empírico-mecanísticos calculados e redução da vida útil do pavimento.

Table 6 -Evolution of mechanistic-empirical calculated $L E F s$ and reduction of pavement serviceable life.

\begin{tabular}{cccc}
\hline $\begin{array}{c}\text { Condições de } \\
\text { carregamento } \\
\text { (kN/eixo) }\end{array}$ & $\begin{array}{c}\text { Pressão de } \\
\text { enchimento kPa } \\
\text { (psi) }\end{array}$ & $\begin{array}{c}\text { FEC } \\
\text { (Calculado) }\end{array}$ & $\begin{array}{c}\text { Redução } \\
\text { da vida } \\
\text { útil (\%) }\end{array}$ \\
\hline $82 \mathrm{kN}$ (Padrão) & $563(80)$ & 1,000 & 0,00 \\
$100 \mathrm{kN}$ (Máxima) & $633(90)$ & 3,166 & 68,41 \\
$120 \mathrm{kN}$ (Excesso) & $703(100)$ & 9,382 & 89,34 \\
\hline
\end{tabular}

Tabela 7-Evolução dos FEC empírico-mecanísticos daAASHTO segundo Pereira (1992) e redução da vida útil do pavimento.

Table 7 - Evolution of the AASHTO mechanistic-empirical LEFs values according to Pereira (1992) and reduction of the pavement serviceable life.

\begin{tabular}{cccc}
\hline $\begin{array}{c}\text { Condições de } \\
\text { carregamento } \\
\text { (kN/eixo) }\end{array}$ & $\begin{array}{c}\text { Pressão de } \\
\text { enchimento kPa } \\
\text { (psi) }\end{array}$ & $\begin{array}{c}\text { FEC } \\
\text { (AASHTO) }\end{array}$ & $\begin{array}{c}\text { Redução } \\
\text { da vida } \\
\text { útil (\%) }\end{array}$ \\
\hline $82 \mathrm{kN}$ (Padrão) & $563(80)$ & 1,000 & 0,00 \\
$100 \mathrm{kN}$ (Máxima) & $633(90)$ & 2.296 & 56,45 \\
$120 \mathrm{kN}$ (Excesso) & $703(100)$ & 5,027 & 80,11 \\
\hline
\end{tabular}

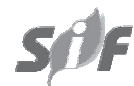

Na Tabela 7, apresenta-se um resumo da redução de vida útil prevista para a estrutura do pavimento estudado, considerando os valores dos FEC empírico-mecanísticos calculados com o expoente médio 4,298 utilizado pela AASHTO, de acordo com Pereira (1992).

Adotou-se a variação do FEC como medida de previsão de vida útil da estrutura do pavimento. A metodologia desta análise está embasada nas considerações apresentadas por Albano (2005).

Considerando os FEC empírico-mecanísticos calculados, com o expoente $b=5,959$ na equação 2 , conforme apresentado na Tabela 6, ao se passar de um N igual 2,55 x $10^{5}$ operações da carga por eixopadrão (carga por eixo igual a $82 \mathrm{kN}$ e pressão de enchimento dos pneus igual a $533 \mathrm{kPa}$ ) para um $\mathrm{N}$ calculado com $100 \mathrm{kN}$ de carga por eixo e pressão de enchimento dos pneus igual a $633 \mathrm{kPa}$ (90 psi), a redução da vida útil prevista para o pavimento foi da ordem de 68,41\%. Do ponto de vista teórico, a vida útil do pavimento diminuiria para 3,42 anos. A mesma análise é aplicada para os demais valores dos fatores de equivalência de carga (FEC) apresentados na Tabela 7.

\subsection{Tensões verticais na superfície do subleito}

Aplicando a equação 2, para um valor de módulo de resiliência de $600 \mathrm{kgf} / \mathrm{cm}^{2}$ obtido pela equação 3 e para um valor de $\mathrm{N}$ igual a 2,55 x $10^{5}$ solicitações do eixo-padrão, obteve-se um valor de tensão vertical admissível $\left(\sigma_{v_{a d m}}\right)$ no topo do subleito de $78,3 \mathrm{kPa}$.

A Tabela 8 apresenta valores de tensões verticais na superfície do subleito, obtidos por meio do programa computacional ELSYM5.

Tabela 8 - Valores das tensões verticais previstas no topo do subleito para espessura da melhoria do subleito de $0,20 \mathrm{~m}$ em função do carregamento.

Table 8 - Values of vertical stress predicted on the subgrade top for a 0.2 m sub-grade improvement layer, in function of the load.

\begin{tabular}{ccc}
\hline $\begin{array}{c}\text { Condições de } \\
\text { carregamento } \\
(\mathrm{kN} / \text { eixo) }\end{array}$ & $\begin{array}{c}\text { Pressão de } \\
\text { enchimento kPa } \\
(\mathrm{psi})\end{array}$ & $\begin{array}{c}\text { Tensão vertical } \\
\text { no topo do } \\
\text { subleito (kPa) }\end{array}$ \\
\hline $82 \mathrm{kN}$ (Padrão) & $563(80)$ & 167 \\
$100 \mathrm{kN}$ (Máxima) & $633(90)$ & 200 \\
$120 \mathrm{kN}$ (Excesso) & $703(100)$ & 235 \\
\hline
\end{tabular}

Revista Árvore, Viçosa-MG, v.35, n.3, p.539-545, 2011 
Analisando os valores da tensão vertical no topo do subleito apresentados na Tabela 8, observou-se que todos os valores superaram a tensão vertical admissível. Isso evidência que a espessura do revestimento primário com a rigidez que lhe foi conferida é insuficiente para suportar a carga do tráfego. Outra análise que ainda se realizou consistiu em determinar qual deveria ser a rigidez da camada do revestimento primário para atender à tensão vertical admissível no topo do subleito, preservando-se a sua espessura de $20 \mathrm{~cm}$ e determinando-se o módulo de elasticidade de $7.500 \mathrm{kgf} / \mathrm{cm}^{2}$ para a referida camada. Esse é um valor difícil de ser alcançado, salvo o emprego de técnicas de estabilização química de solos, por exemplo.

A Tabela 9 contém os resultados das tensões verticais no topo do subleito para uma espessura da camada do revestimento primário de $40 \mathrm{~cm}$.

Analisando os resultados da Tabela 9, observou-se que, com o aumento da espessura da camada de revestimento primário, as tensões verticais no topo do subleito reduziram consideravelmente. No eixo-padrão, a tensão vertical prevista no topo do subleito foi menor do que a tensão admissível. Nos demais carregamentos, as tensões verticais superaram a admissível.

Se fosse utilizado o método empírico do DNIT (2006) para o dimensionamento de pavimentos flexíveis, levando-se em conta os valores CBR contidos na Tabela 2 e um $N=2,55.10^{5}$ operações equivalentes do eixo-padrão, a espessura requerida para proteger o subleito quanto às deformações plásticas excessivas seria de $42 \mathrm{~cm}$. Nesse dimensionamento, considerou-se o material do revestimento primário como granular de coeficiente de equivalência estrutural igual a 1 . Assim, por esse método, se a rigidez da camada do revestimento aumentasse, a sua espessura não sofreria alteração.

Tabela 9 - Valores das tensões verticais previstas no topo do subleito para espessura da melhoria do subleito de $0,40 \mathrm{~m}$ em função do carregamento.

Table 9 - Values of vertical stress predicted on the subgrade top for a $0.4 \mathrm{~m}$ sub-grade improvement layer, in function of the load.

\begin{tabular}{ccc}
\hline $\begin{array}{c}\text { Condições de } \\
\text { carregamento } \\
\text { (kN/eixo) }\end{array}$ & $\begin{array}{c}\text { Pressão de } \\
\text { enchimento kPa } \\
\text { (psi) }\end{array}$ & $\begin{array}{c}\text { Tensão vertical } \\
\text { no topo do } \\
\text { subleito (kPa) }\end{array}$ \\
\hline $82 \mathrm{kN}$ (Padrão) & $563(80)$ & 69 \\
$100 \mathrm{kN}$ (Máxima) & $633(90)$ & 84 \\
$120 \mathrm{kN}$ (Excesso) & $703(100)$ & 100 \\
\hline
\end{tabular}

\section{CONCLUSÕES}

Na análise da determinação do FEC (DNIT, 2006) para eixo simples de rodas duplas em função das deflexões recuperáveis, concluiu-se que o conhecimento do carregamento por eixo e da pressão de enchimento dos pneus da frota comercial que utiliza a via não pavimentada representa, com mais evidência, a realidade das solicitações da estrutura do pavimento do que simplesmente empregar informações sobre o volume médio diário de tráfego misto.

Quanto ao dimensionamento da espessura da camada de revestimento primário (estrutura com duas camadas), tendo em vista os parâmetros de rigidez e deformabilidade adotados para a estrutura e carregamentos apresentados nas Tabelas 1 e 2, verificou-se que:

- Quando se emprega o método empírico do DNIT (2006) para dimensionamento de pavimentos flexíveis, sendo a camada do revestimento primário granular, a sua espessura fica condicionada à rigidez do subleito. Se o suporte do revestimento primário aumentar, não haverá alteração na espessura da referida camada, pois ela não deixará de ser granular, salvo alterações em sua estrutura via técnicas de estabilização química de solos ou mistura de outros materiais capazes de modificar a sua resistência à tração.

- Pela teoria elástica, se houver ganhos de rigidez do revestimento primário, por exemplo, por meio do aumento da energia de compactação, a sua espessura tenderá a diminuir, devido à redução da tensão vertical no topo do subleito. Assim, esse método parece mais adequado para a finalidade pretendida.

\section{AGRADECIMENTOS}

À Fundação de Amparo à Pesquisa do Estado da Bahia (FAPESB), pela concessão da bolsa de pesquisa ao primeiro autor; e ao Departamento de Engenharia Civil da Universidade Federal de Viçosa (DEC/UFV), pelo suporte computacional para a análise dos dados.

\section{REFERÊNCIAS}

\section{ALBANO. J. F. Efeitos dos excessos de} carga sobre a durabilidade de pavimentos. 2005. 211f. Tese (Doutorado em Engenharia Civil) - Programa de Pós Graduação em Engenharia de Produção. Universidade Federal do Rio Grande do Sul, Porto Alegre, 2005. 
AGÊNCIANACIONAL DE TRANSPORTES TERRESTRES - ANTT. Anuário Estatístico dos Transportes Terrestres. Brasília: 2005.

BALBO, J. T. Pavimentação asfáltica: materiais, projeto e restauração. São Paulo: Oficina de Textos, 2007. 558p.

DEPARTAMENTO NACIONAL DE ESTRADAS DE RODAGEM - DNER. Coletânea de Normas. 8v. Determinação das deflexões no pavimento pela viga Benkelman: ME 24/94. Rio de Janeiro: Ministério dos Transportes, 1994. v.1.6p.

\section{DEPARTAMENTO NACIONAL DE INFRA-} ESTRUTURA DE TRANSPORTES - DNIT. Manual de pavimentação. 3.ed. Rio de Janeiro: Instituto de Pesquisas Rodoviárias, 2006. 274p.

PEREIRA, D. R. A. M. Contribuição ao estudo de fatores de equivalência de cargas. 1992. 204p. Dissertação (Mestrado em Engenharia) - Departamento de Engenharia de Transportes. Escola Politécnica da Universidade de São Paulo, São Paulo, 1992.
PINTO, S.; PREUSSLER, E. Pavimentação rodoviária: conceitos fundamentais sobre pavimentos flexíveis. Rio de Janeiro: Copiadora e Artes, 2001. 259p.

SENÇO, W. Manual de técnicas de pavimentação. São Paulo: Pini, 1997. v.1. 746p.

TIMM, D. H.; NEWCOMB, D. E. Lies, damned lies and traffic forecasting, hot mix asphalt technology. Washington: Edition of National Asphalt Pavement Association, 2002. p.14-21.

TRINDADE, T. P. et al. Estudo da durabilidade de misturas solo-rbi grade 81 com vistas à aplicação em estradas florestais e camadas de pavimentos convencionais. Revista Árvore, v.29, n.4, p.592-600, 2005.

VELTEN, R. Z. et al. Caracterização mecânica de misturas solo-escória de alto granulada moída para aplicações em estradas florestais. Revista Árvore, v.30, n.2, p.235-240, 2006.

YODER, E. J.; WITCZAK, M. W. Principles of pavement design. 2.ed. New York: John Wiley \& Sons, 1975. 711p. 